\title{
Antitumoral effect of PLK-1-inhibitor BI2536 in combination with cisplatin and docetaxel in squamous cell carcinoma cell lines of the head and neck
}

\author{
JENS WAGENBLAST, DANIEL HIRTH, ANNE ECKARDT, MARTIN LEINUNG, \\ MARC DIENSTHUBER, TIMO STÖVER and MARKUS HAMBEK
}

ENT Department, Medical School, Goethe University, D-60590 Frankfurt am Main, Germany

Received July 9, 2012; Accepted November 16, 2012

DOI: $10.3892 / \mathrm{mco} .2012 .45$

\begin{abstract}
Inhibition of the polo-like-kinase-1 (PLK-1) has been shown to be effective in several haematological and solid tumor models. In this systemic in vitro study, the antitumor effect of BI2536, a small molecule inhibitor of PLK-1, in combination with cisplatin and docetaxel was examined in nine squamous cell carcinoma cell lines, most of which had a head and neck origin (SCCHN). Dose escalation studies were conducted with nine SCCHN cell lines using BI2536, cisplatin and docetaxel in cell line-specific concentrations. Growth inhibitory and proapoptotic effects were measured quantitatively using cytohistology and a Human Apoptose Array kit. BI2536 in combination with cisplatin and docetaxel showed a markedly higher antiproliferative and apoptotic activity in the SCCHN cell lines investigated ( $\mathrm{P} \leq 0.008)$, compared with single agent cisplatin or docetaxel alone. The findings of this study showed that the addition of PLK-1-inhibitor BI2536 to conventional chemotherapeutic drugs led to a statistically higher antiproliferative and apoptotic effect in SCCHN cell lines compared with cisplatin or docetaxel alone. Inaugurating BI2536 in the clinical setting might enhance the antitumoral activity of conventional drugs, possibly leading to less toxic side effects of cancer therapy.
\end{abstract}

\section{Introduction}

One of the most common malignant diseases worldwide occurs in the head and neck area, with $>600,000$ new diagnoses made worldwide annually (1).

Although therapeutic treatment including surgical techniques and conservative drug strategies have become more

Correspondence to: Dr Jens Wagenblast, ENT Department, University Hospital, Theodor-Stern-Kai 7, D-60590 Frankfurt/Main, Germany

E-mail: jens.wagenblast@kgu.de

Key words: BI2536, cisplatin, docetaxel, squamous cell head and neck carcinoma cell lines, growth inhibition, apoptosis efficient in the last decades, prognosis of this type of cancer remains poor. Identifying novel efficient and well-tolerable treatment strategies with a long-term beneficial effect for these patients is important. Thus, new antineoplastic drugs must be found and evaluated in vitro and in vivo (2).

Polo-like-kinase-1 (PLK-1) has been preclinically validated as a potential cancer target, leading to small-molecule inhibitors of PLK-1 becoming attractive candidates for anticancer drug development (3). At present, five mammalian PLK family members, PLK-1 to -5 , have been identified $(4,5)$. Recent advances in the systemic biology of PLK-1 have greatly improved our understanding of its regulation, target and function. Overexpression of PLK-1 in human tumors and observations on the inhibition of cell proliferation by microinjecting antibodies against PLK-1 into HeLa cells initiated a number of studies aiming to evaluate PLK-1 as a potential target in cancer treatment (6-8). PLK-1 is a key actor of cell cycle at the G2/M transition and in mitosis (9). Furthermore, its overexpression is known to be a negative prognostic marker in certain specific human cancer types $(10,11)$.

BI2536 is an ATP-competitive PLK-1 inhibitor that was identified by screening a diverse library of organic compounds. It causes prometaphase arrest, mitotic abnormalities and apoptosis in a wide range of tumor cell lines $(10,12)$. Notably, BI2536 showed high efficacy in vivo at well-tolerated doses, inducing tumor regression in different xenograft models (10). In a previous systemic in vitro investigation we demonstrated that BI2536 had an eminent antitumoral effect in nine cases of squamous cell carcinoma of the head and neck (SCCHN) (13).

The aim of the present study was to present the antitumoral effects of BI2536, a potent small-molecule inhibitor of mammalian PLK-1, that inhibits PLK-1 activity at low nanomolar concentrations, when combined with cisplatin and docetaxel in nine different tumor cell lines of squamous carcinoma origin.

\section{Materials and methods}

Materials. Nine different squamous carcinoma cell lines, most of them of head and neck origin, were evaluated in this study. A-431 cells were obtained from American Type Culture Collection (Wesel, Germany). PE/CA-PJ 15, 41, 49 and 34 


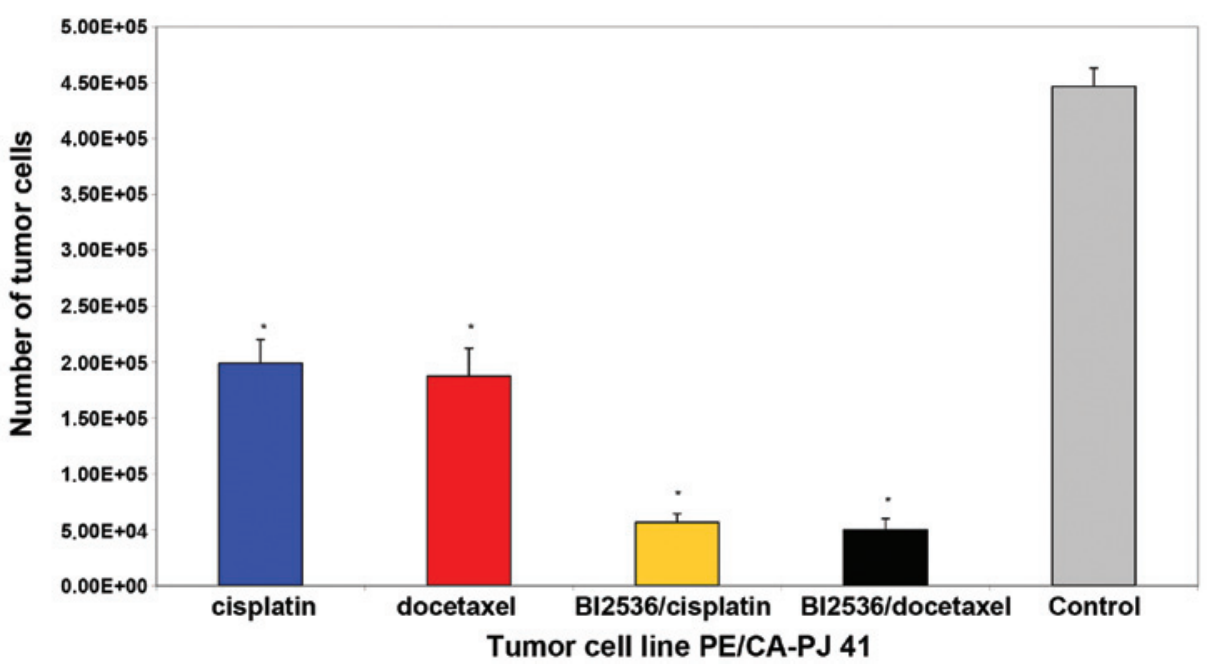

Figure 1. Growth-inhibitory effect of single agent cisplatin (blue column) and docetaxel (red column), as well as the combination of BI2536/cisplatin (yellow column) and BI2536/docetaxel (black column) at a fixed cell line-specific concentration as shown in Table I after $72 \mathrm{~h}$ treatment in PE/CA-PJ 41 cell line. The results of the other cell lines treated were similar to these. The corresponding untreated tumor cell line (grey column) served as the control and was incubated, according to the manufacturer's instructions, only with antibiotics at $37^{\circ} \mathrm{C}$ in the cell type-specific medium Quantum 263 with L-glutamine . The absolute tumor cell numbers in the treated and control cell lines were determined in a Rosenthal chamber at $72 \mathrm{~h}$ after treatment or incubation with Quantum 263 (controls), respectively. Mean values of three independent experiments with standard deviation are shown. Single agent differences vs. combination treatment and untreated controls are indicated by asterisks. Combination treatment of BI2536 with cisplatin- or docetaxel-inhibited cell proliferation significantly higher compared to therapy with cisplatin or docetaxel alone $(\mathrm{P} \leq 0.015)$.

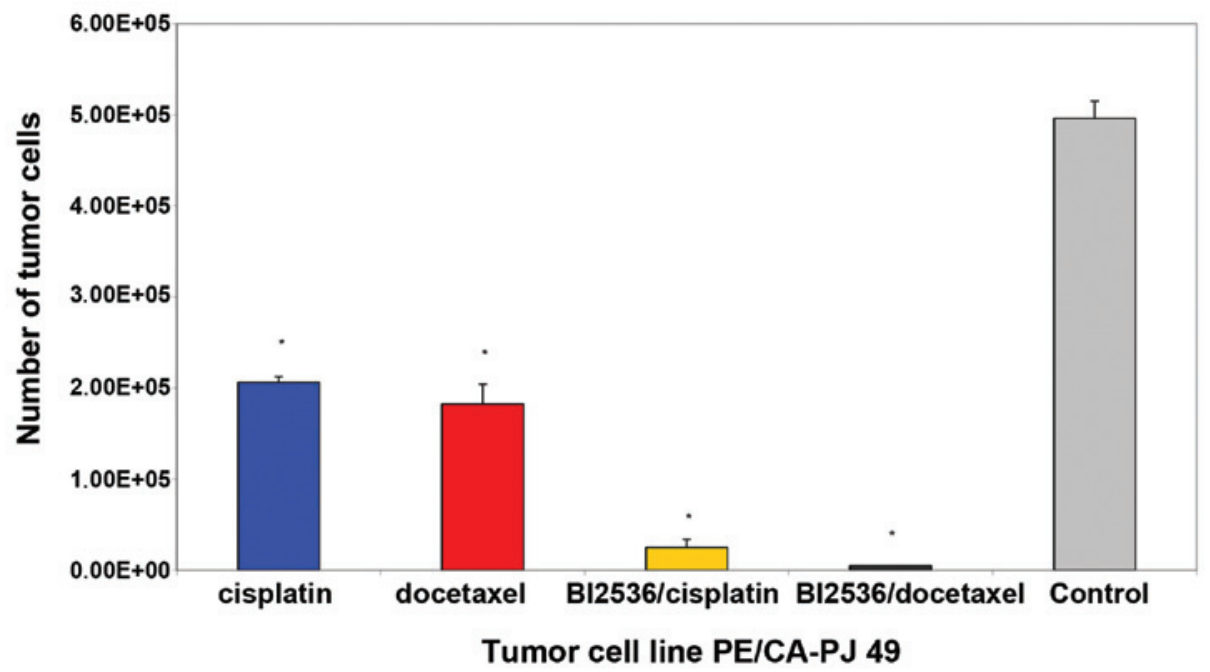

Figure 2. Growth-inhibitory effect of single agent cisplatin (blue column) and docetaxel (red column), as well as the combination of BI2536/cisplatin (yellow column) and BI2536/docetaxel (black column) at a fixed cell line-specific concentration, as shown in Table I after $72 \mathrm{~h}$ treatment in PE/CA-PJ 49 cell line. The results of the other cell lines treated were similar to these. The corresponding untreated tumor cell line (grey column) served as the control and was incubated, according to the instructions of the suppliers, only with antibiotics at $37^{\circ} \mathrm{C}$ in the cell type-specific medium Quantum 263 with L-glutamine. The absolute tumor cell numbers in treated and control cell lines were determined in a Rosenthal chamber at $72 \mathrm{~h}$ after treatment or incubation with Quantum 263 (controls), respectively. Mean values of three independent experiments with standard deviation are shown. Single agent differences vs. combination treatment and untreated controls are indicated by asterisks. Combination treatment of BI2536 with cisplatin or docetaxel inhibited cell proliferation significantly higher than therapy with cisplatin or docetaxel alone $(\mathrm{P} \leq 0.015)$.

cells were obtained from European Collection of Cell Cultures (Sigma Aldrich, Taufkirchen, Germany), while Cal-27 and Kyse-140 cells from DSMZ GmbH (Braunschweig, Germany).

CLS-354 and UM-SCC-14C cells were obtained from CLS

Cell Lines Service (Eppelheim, Germany). The fibroblast cell line was a gift from the Department of Dermatology of the University Hospital, Frankfurt/Main, Germany.

BI2536 was provided by Boehringer Ingelheim $\mathrm{GmbH}$, Austria. Squamous carcinoma cell lines were cultivated according to the instructions of the suppliers, with antibiotics at $37^{\circ} \mathrm{C}$ in the cell type-specific medium Quantum 263 with L-glutamine (PAA Laboratories GmbH, Cölbe, Germany).

Methods. Cells were seeded in 96-multiwell plates $\left(1 \times 10^{5}\right.$ cells/well), and following $24-\mathrm{h}$ incubation the cells were treated with cisplatin or docetaxel alone or in combination with BI2536 for 24, 48 and $76 \mathrm{~h}$, respectively. In the experiments described in this study, BI2536 as well as 


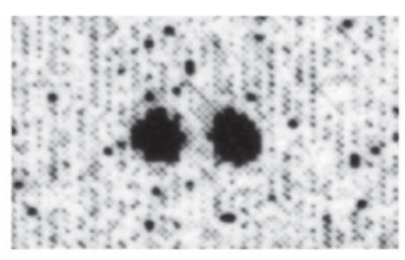

Positive Control

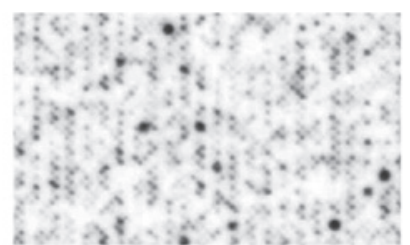

Negative Control

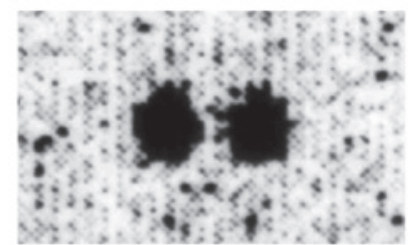

Pro-Caspase-3

Figure 3. Detection of pro-caspase-3 as a typical apoptosis marker by Human Apoptose Array kit. Besides other cell markers (data not shown) we detected the typical apoptosis marker pro-caspase-3 in nine different squamous carcinoma cell lines treated with BI2536 in combination with cisplatin and docetaxel. Fig. 2 shows the results received when treating PE/CA-PJ 49 cells with BI2536 in combination with cisplatin and docetaxel.

Table I. Cell line-specific drug concentration of cisplatin and docetaxel used in our experiments.

\begin{tabular}{lcc}
\hline Tumor cell line & $\begin{array}{c}\text { Concentration } \\
\text { of cisplatin }(\mu \mathrm{M})\end{array}$ & $\begin{array}{c}\text { Concentration } \\
\text { of docetaxel }(\mathrm{nM})\end{array}$ \\
\hline PE/CA-PJ 15 & 1.56 & 2.5 \\
PE/CA-PJ 34 & 1.56 & 2.5 \\
PE/CA-PJ 41 & 0.19 & 5.0 \\
PE/CA-PJ 49 & 1.56 & 15.0 \\
CLS 354 & 1.56 & 10.0 \\
UM-SCC-14 C & 1.56 & 10.0 \\
Cal-27 & 0.39 & 15.0 \\
Kyse-140 & 3.125 & 5.0 \\
A-431 & 0.78 & 15.0 \\
\hline
\end{tabular}

cisplatin and docetaxel were used in each cell line at a fixed, cell line-specific concentration that had produced maximum growth inhibition in previous systematic investigations in our laboratory. The concentration for the cell line investigations was $2.5 \mathrm{nmol} / 1$ for BI2536, showing maximal growth inhibition in our previous dose escalation studies (unpublished data). Doses of cisplatin and docetaxel ranged from 0.19 to 3.125 and 2.5 to $15 \mathrm{nM}$, respectively (Table I).

The number of cells was determined by counting cells in a Rosenthal chamber at 24, 48 and $72 \mathrm{~h}$ after treatment. Apoptosis was detected by microscopic cytohistology as well as by the Human Apoptose Array kit (R\&D Systems, Wiesbaden, Germany), as previously described by other investigators $(14,15)$.

Each experiment was carried out in triplicate. For statistical analysis, a Wilcoxon test for matched pairs (depending on samples) was performed using the SPSS 19.0 software for Windows. $\mathrm{P}<0.05$ was considered to indicate a statistically significant difference.

\section{Results}

Combination of cisplatin and doxetaxel yields higher proliferation activity. Nine different squamous carcinoma cell lines, most of them of head and neck origin, were tested in this study. Following 24-h incubation, the cells were treated with cisplatin and docetaxel alone or in combination with BI2536 for 24, 48 and $76 \mathrm{~h}$, respectively. Compared with the untreated control groups, the PLK-1-inhibitor BI2536 as well as cisplatin and docetaxel had a highly significant antiproliferative and apoptotic effect used as single agent treatment in the nine tumor cell lines $(\mathrm{P}=0.008)$. The drug combination of $\mathrm{BI} 2536$ and cisplatin showed a markedly higher antiproliferative activity compared with cisplatin alone $(\mathrm{P}<0.008)$. The same phenomenon was detected in the combination of BI2536 and docetaxel compared to docetaxel monotherapy $(\mathrm{P}=0.008)$. Figs. 1 and 2 show these results in two different tumor cells lines representative for the nine tumor cell lines investigated.

Detection of pro-caspade-3 as a typical apoptosis marker. Apoptosis was detected by microscopic cytohistology as well as by the Human Apoptose Array kit (R\&D Systems), detecting pro-caspase-3 as a typical molecular apoptosis marker (Fig. 3).

Our findings indicate that the addition of PLK-1 inhibitor BI2536 to conventional drugs, such as cisplatin and docetaxel, led to a significantly higher antiproliferative effect compared to a single agent treatment with cisplatin or docectaxel alone.

\section{Discussion}

Therapy-associated side effects in the chemotherapeutical treatment of head and neck cancer disease have led to a concentrated search for novel, well-tolerable therapeutic agents. Although cisplatin is a potent and widely used anticancer drug, in solid tumors, its clinical use is often limited by hematologic toxicity, nephrotoxicity, myelosuppression as well as other complications, such as cardiovascular consitions and hearing loss $(16,17)$. The group of taxanes is also highly active in head and neck cancer disease. Single-agent taxane therapy may have a response rate between 20 and $40 \%$. Serious therapy-associated toxic side effects are neutropenia, pulmonary infection, mucositis followed by chronic dysphagia and weight loss. As a combination chemotherapy taxanes and cisplatin together have yielded promising response rates ranging from 40 to $70 \%$ in patients with recurrent or metastatic SCCHN (18).

Identification of innovative antineoplastic chemotherapeutical drugs with reduced or well-tolerable side effects and less toxicity, but with relevant clinical activity is a major challenge in modern oncology. Although surgical and non-surgical treatment modalities of head and neck cancer have become more sophisticated over the last decades, the 5-year overall survival rate in stage UICC III and IV remains poor (19). Previous 
biochemical investigations have led to a better understanding of biological tumor behaviour, thereby improving treatment strategies. PLK-1 is known to be essential in cell cycle regulation through functioning in centrosome maturation, spindle formation, mitotic entry and cytokinesis. Elevated PLK-1 levels have been found in several human cancers, including breast and colorectal cancer, neuroblastoma, rhabdomyosarcoma and squamous cell carcinoma (20-23). Previous studies have demonstrated that the PLK-1 level correlates with metastasis in cervical lymph nodes. This emphasizes that determination of PLK expression in primary tumors of the head and neck may be a marker of the metastatic potential of this tumor identity (24). Head and neck cancer patients are likely to be prospectively classified on the basis of the PLK expression level of their tumor with regard to better or worse prognosis, suggesting PLK expression to be a discrimination marker.

BI2536 is a first-class PLK-1 inhibitor. It is an ATP-competitive kinase inhibitor that suppresses the enzymatic activity of PLK-1 and shows over 1,000-fold selectivity for PLK-1 against a large panel of various tyrosine and serine/threonine kinases $(22,25)$. With regard to results of dose-escalating Phase I studies, BI2536 was well tolerated (25). Despite the fact that in clinical Phase I and II trials BI2536 was administered in various tumor entities, further in vitro studies focusing on the biological and pharmacological profile of BI2536 need to be conducted (26).

To the best of our knowledge, the findings of our systemic in vitro study have demonstrated, for the first time, the growth-inhibitory and proapoptotic effects of BI2536 in combination with cisplatin and docetaxel in squamous cell carcinoma cell lines.

As the addition of BI2536 to chemotherapy with cisplatin and docetaxel enhanced the antiproliferative effect of the two cytotoxic agents, this may offer the opportunity to reduce the doses of cisplatin and docetaxel, thereby improving the safety and tolerance of cisplatin- and docetaxel-based chemotherapy in the clinical routine. Notably, treatment of SCCHN cell lines with BI2536 in combination with cisplatin and docetaxel induced a strong inhibition of cell proliferation and significantly enhanced apoptosis compared to single agent treatment with cisplatin or docetaxel alone.

Although the impact of combination chemotherapy is considered controversial in the present literature, our findings suggest that BI2536 improves the antineoplastic activity of combination drug regimes. Taken together, based on our promising in vitro results and previous studies in this field, we conclude that the addition of BI2536 to cisplatin and docetaxel may improve the efficacy of chemotherapeutical treatment in patients with head and neck cancer, as well as allow for the use of cisplatin and docetaxel at reduced doses in the future without compromising the antitumor effects. Future clinical studies should be conducted to prove this hypothesis.

\section{Acknowledgements}

The authors thank Boehringer Ingelheim $\mathrm{GmbH}$ for providing BI2536 free of charge. The authors also thank Erika Weith for her excellent technical support.

\section{References}

1. Mehanna H, Paleri V, West CM and Nutting C: Head and neck cancer - Part 1: Epidemiology, presentation, and prevention. BMJ 341: c4684, 2010.

2. Wagenblast J, Hambek M, Baghi M, Gstöttner W, Strebhardt K, Ackermann $\mathrm{H}$ and Knecht R: Antiproliferative activity of bortezomib alone and in combination with cisplatin or docetaxel in head and neck squamous cell carcinoma cell lines. J Cancer Res Clin Oncol 134: 323-330, 2008.

3. Strebhardt K: Multifaceted polo-like kinases: drug targets and antitargets for cancer therapy. Nat Rev Drug Discov 9: 643-660, 2010.

4. Barr FA, Sillje HH and Nigg EA: Polo-like kinases and the orchestration of cell division. Nat Rev Mol Cell Biol 5: 429-440, 2004.

5. Andrysik Z, Bernstein WZ, Deng L, Myer DL, Li YQ, Tischfield JA, et al: The novel mouse Polo-like kinase 5 responds to DNA damage and localizes in the nucleolus. Nucleic Acids Res 38: 2931-2943, 2010.

6. Holtrich U, Wolf G, Bräuninger A, Karn T, Böhme B, Rübsamen-Waigmann $\mathrm{H}$ and Strebhardt K: Induction and downregulation of PLK, a human serine/threonine kinase expressed in proliferating cells and tumors. Proc Natl Acad Sci USA 91: 1736-1740, 1994.

7. Lane HA and Nigg EA: Antibody microinjection reveals an essential role for human polo-like kinase 1 (Plk1) in the functional maturation of mitotic centrosomes. J Cell Biol 135: 1701-1713, 1996.

8. Spankuch B, Steinhauser I, Wartlick H, Kurunci-Csacsko E, Strebhardt KI and Langer K: Downregulation of Plk1 expression by receptor-mediated uptake of antisense oligonucleotide-loaded nanoparticles. Neoplasia 10: 223-234, 2008.

9. Van de Weerdt BC and Medema RH: Polo-like kinases: a team in control of the division. Cell Cycle 5: 853-864, 2006.

10. Steegmaier M, Hoffmann M, Baum A, Lénárt P, Petronczki M, Krssák M, et al: BI 2536, a potent and selective inhibitor of polo-like kinase 1, inhibits tumor growth in vivo. Curr Biol 17: 316-322, 2007.

11. Takai N, Hamanaka R, Yoshimatsu J and Miyakawa I: Polo-like kinases (Plks) and cancer. Oncogene 24: 287-291, 2005.

12. Lénárt P, Petronczki M, Steegmaier M, Di Fiore B, Lipp JJ, Hoffmann M, et al: The small-molecule inhibitor BI 2536 reveals novel insights into mitotic roles of polo-like kinase 1. Curr Biol 17: 304-315, 2007.

13. Wagenblast J, Hirth D, Thron L, Arnoldner C, Diensthuber M, Stöver T and Hambek M: Effects of the Polo-like-kinase-1inhibitor BI2536 in squamous cell carcinoma cell lines of the head and neck. Oncol Lett 4: 175-177, 2012.

14. Datta D, Banerjee P, Gasser M, Waaga-Gasser AM and Pal S: CXCR3-B can mediate growth-inhibitory signals in human renal cancer cells by down-regulating the expression of heme oxygenase-1. J Biol Chem 285: 36842-36848, 2010.

15. Ray RB, Raychoudhuri A, Steele R and Nerurkar P: Bitter melon (Momordica charantia) extract inhibits breast cancer cell proliferation by modulating cell cycle regulatory genes and promotes apoptosis. Cancer Res 70: 1925-1931, 2010.

16. Forastiere AA, Leong T, Rowinsky E, Murphy BA, Vlock DR, DeConti RC and Adams GL: Phase III comparison of high-dose paclitaxel + cisplatin + granulocyte colony-stimulating factor versus low-dose paclitaxel + cisplatin in advanced head and neck cancer: Eastern Cooperative Oncology Group Study E1393. J Clin Oncol 19: 1088-1095, 2001.

17. Schrijvers D, Johnson J, Jiminez U, Gore M, Kosmidis P, Szpirglas H, et al: Phase III trial of modulation of cisplatin/ fluorouracil chemotherapy by interferon alfa-2b in patients with recurrent or metastatic head and neck cancer. Head and Neck Interferon Cooperative Study Group. J Clin Oncol 16: 1054-1059, 1998.

18. Kohno N: The role of taxanes for head and neck cancer. Gan To Kagaku Ryoho 32: 2035-2039, 2005 (In Japanese).

19. Jemal A, Siegel R, Ward E, Murray T, Xu J and Thun MJ: Cancer statistics. CA Cancer J Clin 57: 43-66, 2007.

20. Ackermann S, Goeser F, Schulte JH, Schramm A, Ehemann V, Hero B, et al: Polo-like kinase 1 is a therapeutic target in high-risk neuroblastoma. Clin Cancer Res 17: 731-741, 2011.

21. Hu K, Lee C, Qiu D, Fotovati A, Davies A, Abu-Ali S, et al: Small interfering RNA library screen of human kinases and phosphatases identifies polo-like kinase 1 as a promising new target for the treatment of pediatric rhabdomyosarcomas. Mol Cancer Ther 8: 3024-3035, 2009. 
22. Chopra P, Sethi G, Dastidar SG and Ray A: Polo-like kinase inhibitors: an emerging opportunity for cancer therapeutics. Expert Opin Investig Drugs 19: 27-43, 2010.

23. Knecht R, Oberhauser C and Strebhardt K: PLK (polo-like kinase), a new prognostic marker for oropharyngeal carcinomas. Int J Cancer 89: 535-536, 2000.

24. Knecht R, Elez R, Oechler M, Solbach C, von Ilberg C and Strebhardt K: Prognostic significance of polo-like kinase (PLK) expression in squamous cell carcinomas of the head and neck. Cancer Res 59: 2794-2797, 1999.

25. Mross K, Frost A, Steinbild S, Hedbom S, Rentschler J, Kaiser R, et al: Phase I dose escalation and pharmacokinetic study of BI 2536, a novel Polo-like kinase 1 inhibitor, in patients with advanced solid tumors. J Clin Oncol 26: 5511-5517, 2008.
26. Schöffski P, Blay JY, De Greve J, Brain E, Machiels JP, Soria JC, et al: Multicentric parallel phase II trial of the polo-like kinase 1 inhibitor BI 2536 in patients with advanced head and neck cancer, breast cancer, ovarian cancer, soft tissue sarcoma and melanoma. The first protocol of the European Organization for Research and Treatment of Cancer (EORTC) Network Of Core Institutes (NOCI). Eur J Cancer 46: 2206-2215, 2010. 